\title{
Spiralian Model Systems
}

\author{
Guest Editor
}

\author{
Jonathan Q. Henry
}

University of Illinois, Dept. Cell E Developmental Biology, Urbana, IL, USA

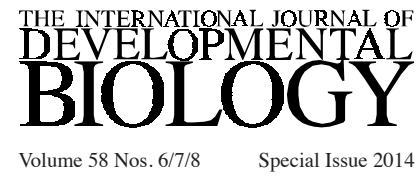





\section{Preface}

\section{Contemporary Spiralian Developmental Biology}

This current issue of The International Fournal of Developmental Biology focuses attention on many models that represent the metazoan super-clade called the Spiralia (Lophotrochozoa). This clade encompasses the largest number of metazoan phyla, with arguably the most diverse array of animal body plans. Because of this, I choose to focus attention mainly on members that exhibit the highly conserved early pattern of development that involves spiral cleavage (e.g., annelids, molluscs, nemerteans). This clade includes other phyla, such as the platyhelminthes (e.g., planaria, which were the subject of a previous Special Issue of the Int. F. Dev. Biol., Saló and Agata, 2012), as well as brachiopods, bryozoans, and many others that contain species that do not exhibit spiral cleavage. Together, this group is very important to our understanding of the evolution of the Metazoa and the fundamental developmental processes that generate animal body plans. Surprisingly, it has received much less attention compared to the other major clades of bilaterians: the deuterostomes and ecdysozoans. As evidence of this fact, there have not been any comparable collections of work that highlight the Spiralia, with one notable exception that recently appeared in the journal Integr. Comp. Biol. (Lambert and Seaver, 2010). It is hoped that this Special Issue will not only showcase work that has taken place, but convince readers that this group deserves much more attention.

Developmental studies using spiralians began in earnest in the late 1800s, with work being conducted at major marine laboratories, such as the Marine Biological Laboratory in Woods Hole, MA, USA. These earlier studies played a pivotal role in understanding the nature of early development and addressed important issues related to the organization of the egg and embryonic cells, and the extent to which development proceeds from preformed patterns vs. epigenetic mechanisms. Those studies, particularly ones that deciphered and compared embryonic cell lineages, also contributed to discussions of Haeckel's "Biogenetic Law" and our understanding of the connections between ontogeny and phylogeny (Maienschein, 1978). In fact, that early research should be considered as an important point in the birth of evolutionary-developmental biology. Since that time, spiralian model systems have been developed and used to study many important questions in the fields of developmental biology, neurobiology, evolution and others, which are described in the articles included in this Special Issue of The Int. F. Dev. Biol. Each highlights different species being used as key models, and the vast scope of questions that are being addressed using those systems.

I am very grateful to Richard Behringer whose idea it was to focus attention on this group of organisms and who suggested that I serve as the Guest Editor of this Special Issue. I am also grateful to Juan Aréchaga for all his help and encouragement during the course of its production. I especially thank all of the authors who contributed to this issue. Together they have assembled a timely collection of work that reveals the diversity and significance of this clade. Their effort and passion will inspire future generations to continue work in these fields. It was a real pleasure to assemble this Special Issue, and to see the exciting work that has been performed. In fact, this collection of articles only covers a part of the research undertaken with spiralian model systems. I would also like to thank the many anonymous reviewers who gave their precious time to evaluate these manuscripts. Finally, I thank the Editorial Team of The International Fournal of Developmental Biology for all of their hard work in assembling this issue.

Fonathan Henry

Urbana, Illinois, October 2014

\section{References}

MAIENSCHEIN, J. (1978). Cell lineage, ancestral reminiscence, and the biogenetic law. J Hist Biol 11: 129-158.

SALÓ, M. and AGATA, K. (2012). Planarian regeneration: a classic topic claiming new attention. Int. J. Dev. Biol. 56: 1-4

SALÓ, M. and AGATA, K. [Eds.] (2012). The Planaria Model System. Int J Dev Biol 56: 1-196.

LAMBERT, D. and SEAVER, E. [Eds.](2010). Spiralian Development: Conservation and Innovation. Integr. Comp. Biol. 50: 695-777.

Int. J. Dev. Biol. 58: 385-387 (2014)

doi: 10.1387/ijdb.140289jh 